\title{
Diagnosis of viral hepatitis
}

\author{
Philippa J. Easterbrook ${ }^{\mathrm{a}}$, Teri Roberts ${ }^{\mathrm{b}}$, Anita Sands $^{\mathrm{c}}$, and Rosanna Peeling ${ }^{\mathrm{d}}$
}

\begin{abstract}
Purpose of review
Chronic hepatitis $B$ virus (HBV) and hepatitis $C$ virus (HCV) infections and HIV-HBV and HCV coinfection are major causes of chronic liver disease worldwide. Testing and diagnosis is the gateway for access to both treatment and prevention services, but there remains a large burden of undiagnosed infection globally. We review the global epidemiology, key challenges in the current hepatitis testing response, new tools to support the hepatitis global response (2016-2020 Global Hepatitis Health Sector strategy, and 2017 WHO guidelines on hepatitis testing) and future directions and innovations in hepatitis diagnostics.
\end{abstract}

\section{Recent findings}

Key challenges in the current hepatitis testing response include lack of quality-assured serological and low-cost virological in-vitro diagnostics, limited facilities for testing, inadequate data to guide country-specific hepatitis testing approaches, stigmatization of those with or at risk of viral hepatitis and lack of guidelines on hepatitis testing for resource-limited settings. The new Global Hepatitis Health Sector strategy sets out goals for elimination of viral hepatitis as a public health threat by 2030 and gives outcome targets for reductions in new infections and mortality, as well as service delivery targets that include testing, diagnosis and treatment. The 2017 WHO hepatitis testing guidelines for adults, adolescents and children in low-income and middleincome countries outline the public health approach to strengthen and expand current testing practices for viral hepatitis and addresses who to test (testing approaches), which serological and virological assays to use (testing strategies) as well as interventions to promote linkage to prevention and care.

\section{Summary}

Future directions and innovations in hepatitis testing include strategies to improve access such as through use of existing facility and community-based testing opportunities for hepatitis testing, near-patient or pointof-care assays for virological markers (nucleic acid testing and HCV core antigen), dried blood spot specimens used with different serological and nucleic acid test assays, multiplex and multi-disease platforms to enable testing for multiple analytes/pathogens and potential self-testing for viral hepatitis.

\section{Keywords}

dried blood spot, Global Hepatitis Health Sector strategy, hepatitis B virus, hepatitis C virus, nucleic acid amplification test, rapid diagnostic test, WHO testing guidelines

\section{INTRODUCTION}

\section{Epidemiology and burden}

HIV, hepatitis B (HBV) and hepatitis C virus (HCV) infections are major global public health problems, with overlapping modes of transmission and affected populations. It is estimated that 36.9 million people are living with HIV [1,2], 248 million with chronic HBV (CHB) infection [defined as persistence of hepatitis B surface antigen (HBsAg) for 6 months or more] [3"'] and 110 million persons are $\mathrm{HCV}$ antibody-positive, of which 80 million have viraemic $\mathrm{HCV}$ infection [4]. However, the estimates commonly cited in the published literature vary widely [5]. Hepatitis B virus and HCV infections are associated with significant morbidity and mortality due to chronic liver disease. They account for approximately $57 \%$ of cirrhosis and $78 \%$ of hepatocellular carcinoma (HCC) cases worldwide, and together they cause an estimated 1.4 million deaths annually [6]. The burden of HBV and HCV is

\footnotetext{
${ }^{a}$ Global Hepatitis Programme, HIV Department, World Health Organization, ${ }^{b}$ Médecins Sans Frontières, 'Department of Essential Medicines and Health Products, World Health Organization, Geneva, Switzerland and 'London School of Hygiene and Tropical Medicine, London, UK

Correspondence to Philippa J. Easterbrook, Global Hepatitis Programme, HIV Department, World Health Organization, Avenue Appia 20, 1211 Geneva 27, Switzerland. Tel: +41 22791 4518; e-mail: easterbrookp@who.int

Curr Opin HIV AIDS 2017, 12:302-314

DOI: $10.1097 / \mathrm{COH} .0000000000000370$

This is an open access article distributed under the terms of the Creative Commons Attribution-Non Commercial License 4.0 (CCBY-NC), where it is permissible to download, share, remix, transform, and buildup the work provided it is properly cited. The work cannot be used commercially without permission from the journal.
} 


\section{KEY POINTS}

- There are an estimated 2.73 and 2.28 million persons with HIV-hepatitis B surface antigen and HIV-anti-HCV coinfection globally. The greatest burden of HIV-HBV coinfection is in sub-Saharan Africa (71\% of all cases), and for HIV-HCV is in Eastern Europe and central Asia ( $27 \%$ of all cases). The majority of HIV-HCV coinfection (59\% of cases) are in persons who inject drugs.

- The majority of HBV infections are acquired perinatally or in early childhood, many years before HIV acquisition, in contrast to $\mathrm{HCV}$, which is acquired generally in adulthood.

- The current low rate of viral hepatitis testing is a major barrier to scale-up of access to prevention, care and treatment.

- Current viral hepatitis testing strategies include initial serological testing with assays in either rapid diagnostic test or laboratory-based immunoassay format followed by virological testing for HBV DNA and HCV RNA to guide treatment decisions.

- Future directions and innovations in testing include simplified diagnostic algorithms, point-of-care virological assays, multi-disease platforms and self-testing for anti-HCV.

disproportionately high in low-income and middle-income countries (LMICs), particularly in Asia and Africa. Worldwide, the majority of persons with $\mathrm{CHB}$ were infected at birth or in early childhood, and perinatal or horizontal transmission predominates in sub-Saharan Africa (SSA) and Asia. In contrast, in high-income settings, such as North America and Europe, transmission is predominantly via injection drug use and highrisk sexual behaviours, especially in MSM [7]. In middle-income and high-income countries, most $\mathrm{HCV}$ infections occur among persons who inject drugs (PWID), whereas in low-income settings, HCV infection is most commonly associated with unsafe injection practices and procedures in healthcare facilities with inadequate infection control [8].

\section{HIV-hepatitis B virus and hepatitis C virus coinfection}

As HIV-infected persons continue to live longer due to increased uptake of antiretroviral therapy (ART), liver disease has emerged as a leading cause of morbidity and mortality in HIV-infected persons coinfected with HBV or HCV [9-14]. Two recent complementary systematic reviews and meta-analyses have provided updated regional estimates of HIV-HBV and HIV-HCV coinfection across five different population groups [15"',16"'].

There is an estimated global hepatitis B surfance antigen (HBsAg) prevalence of $7.4 \%$ [interquartile range (IQR) 5.0-11.2\%] in HIV-infected persons, and a burden of 2.73 million (IQR 1.7-4.3 million; IQR 1.3-4.4 million) HIV-HBsAg coinfected persons, based on a systematic review [15"']. The greatest burden is in SSA (71\% of all cases; 1.96 million), as it is the most affected region for HIV infection $(65 \%$ or 22 million of total global HIV infections), followed by Southeast Asia ( $10 \%$ of all cases; 288816 ). There is an estimated global HCV antibody (anti-HCV) seroprevalence of $6.2 \%$ [IQR 3.4-11.9 (IQR 5.0-11.2\%)] in HIV-infected persons, and a burden of 2.28 million (IQR 1.3-4.4 million) HIV-HCV antibody coinfected persons [16"'], of which 1.36 million are in PWID. However, in contrast to HIV-HBV infection, the greatest HIV-HCV burden is in Eastern Europe and Central Asia (27\% of all cases), because of the large HIV-infected population of PWID, followed by SSA (19\% of all cases). These recent global estimates of HIV-HBV and HCV coinfection are lower than the previously reported prevalence of coinfection [5,9,17-20].

\section{Differences between epidemiology of HIV- hepatitis $B$ virus and HIV-hepatitis $C$ virus coinfection}

The two companion reviews [15"',16"'] highlight further important distinctions between the epidemiology of HIV-HBV and HIV-HCV coinfection. Prevalence of HCV coinfection varies widely and is highest in PWID [82.4\% (95\% confidence interval (CI) 55.2-88.5)], followed by MSM 6.4\% (3.2-10.0), with much lower rates within the general population [2.4\% (IQR 0.8-5.8)]. In contrast, HBsAg prevalence is similar across different HIV-infected population/exposure groups, and in particular between the general population (6.6\%) and higher risk behaviour groups such as PWID (7.0\%) and MSM (6.1\%). This overall lower HBsAg prevalence, even in very high-risk groups, is largely because the majority of $\mathrm{HBV}$ infection is acquired perinatally or in early childhood, many years before HIV acquisition, in contrast to HCV, which is acquired generally in adulthood, as for HIV. Globally, PWID accounted for only $7 \%$ of all HIV-HBV but $59 \%$ of HIV-HCV coinfections.

\section{Impact of HIV coinfection on natural history of hepatitis $B$ virus and hepatitis $C$ virus}

The interaction with HIV affects the transmission and natural history of HBV and HCV infections $[9,10,12,18]$. HBV coinfection in HIV-infected 
persons is associated with less spontaneous clearance of HBsAg, higher rates of chronicity and occult HBV (HBV DNA detected in the absence of HBsAg), accelerated fibrosis progression with increased risk of cirrhosis and HCC and higher liver-related mortality $[9,10,14,18]$. HIV infection also doubles the risk of $\mathrm{HCV}$ perinatal transmission in HIV-infected mothers [21,22] and is associated with less-spontaneous HCV clearance, higher HCV viral loads and more rapid HCV disease progression [8,23-25]. Although ART improves outcomes in both and HCV coinfected patients, they may also increase the risk of hepatoxicity from ART three-fold to five-fold $[8,10]$, and cross-resistance between HIV and HBV drugs is common [26].

\section{Strategies for control}

\section{Hepatitis $B$ virus}

Universal infant and birth-dose hepatitis B vaccination remains the key strategy for prevention and control of the HBV epidemic [27]. High levels of coverage have resulted in a substantial decrease in new cases of HBV infection in those aged under 15 years [28]. However, rates of adult vaccination in vulnerable high-risk groups, such PWID, MSM, sex workers or prisoners, remain low, and these need to be specifically targeted for hepatitis $\mathrm{B}$ and $\mathrm{C}$ testing, catch up hepatitis B vaccination and other preventive interventions [29]. As Tenofovir (TDF) is the most effective drug for long-term treatment of HBV [30], the impressive global scale-up of HIV testing and treatment [1] together with the increasing adoption of a TDF-based regimen as the preferred first-line ART in adults and children aged more than 3 years [31] represents a major global opportunity to identify and effectively treat those who also have HBV coinfection, as well as to reduce mother-to-child transmission.

\section{Hepatitis C virus}

The new era of highly curative short-course directacting oral antiviral therapies for HCV has revolutionized the treatment of chronic HCV infection and offers the potential to not only improve individual clinical outcomes but also reduce transmission [32]. There is a need to continue to scaleup HIV and HCV prevention interventions in higher risk populations, especially PWID with needle or syringe exchange programmes, opiate substitution therapy and provision of ART. This is in addition to strategies to improve injection safety both worldwide and especially in Eastern Europe and Southeast Asia.

\section{GOALS OF HEPATITIS TESTING}

Testing and diagnosis of $\mathrm{CHB}$ and $\mathrm{HCV}$ infection is the entry point for access to both prevention as well as care and treatment services. Early identification of persons with $\mathrm{CHB}$ or HCV infection enables them to receive the necessary care and treatment to prevent or delay progression of liver disease. Knowledge of HBV status is important to guide optimal selection of ART regimens that include use of TDF, but also in maintaining an agent active against HBV when a treatment switch is required as withdrawal can be associated with liver flares and acute on chronic liver disease [33]. Testing also provides an opportunity to link to interventions that reduce transmission, such as counselling on risk behaviours and the provision of prevention commodities (such as sterile needles and syringes) and hepatitis $B$ vaccination.

\section{CHALLENGES IN HEPATITIS TESTING}

Despite the significant burden of disease due to HBV and HCV infections and HIV-HBV and HCV coinfection, and the advances and opportunities for treatment, the majority of people coinfected with $\mathrm{HBV}$ or HCV remain undiagnosed and unaware of their infection [34]. It is estimated that less than 5\% of people with $\mathrm{CHB}$ or $\mathrm{HCV}$ infection know their status [34,35"'], but in low-income and middleincome settings, this is even lower $(<1 \%)$. This is also particularly poor in populations such as PWID, prisoners, sex workers and MSM, for whom access to care and treatment are already challenging [32].

There are several key reasons for this low rate of hepatitis testing [36]. These include lack of awareness about hepatitis $\mathrm{B}$ and $\mathrm{C}$ disease and potential treatments among patients and healthcare workers; limited facilities or services for hepatitis testing, including those that can reach most affected and vulnerable populations, such as harm reduction services; limited access to reliable and low-cost HBV and HCV diagnostics, including rapid diagnostic tests (RDTs) and virological assays that are quality assured by stringent regulatory authorities and/or WHO prequalified; poor laboratory capacity and limited access to additional tests to stage liver disease and guide treatment decisions, such as transient elastography (e.g. FibroScan); weak hepatitis surveillance programmes and limited national and subnational data on epidemiological situation to guide country-specific hepatitis testing approaches; high levels of stigma, discrimination and social marginalization of those with or at risk of viral hepatitis, such as PWID, MSM, prisoners and sex workers; lack of testing guidance for low-income and middle-income settings and a limited evidence 
base to guide hepatitis testing approaches; lack of political and financial commitment, including from traditional donors, and that few countries have designated hepatitis units and budgets within their health ministries to lead the development of a hepatitis strategy.

\section{Testing strategies}

\section{Hepatitis $B$ virus testing}

CHB infection is diagnosed by detection of HBsAg using serological assays [in either RDT or laboratorybased immunoassay formats that include the enzyme immunoassay (EIA), chemiluminescence immunoassay (CLIA) and electrochemiluminescence assay (ECL)] [37"']. Confirmation of the presence of HBsAg may be carried out by performing a neutralization step using a specific anti-HBs-containing reagent in the same assay or by repeating HBsAg testing using a different assay of similar sensitivity (i.e. two-assay serological testing strategy). The choice of which format of serological assays to use will depend on a variety of factors most importantly, ease of use and the characteristics of the testing site, such as storage facilities, infrastructure, level of staff skills and cost. This is followed by measurement of HBV DNA levels and liver function which, together with staging of liver disease (based on clinical assessment or use of noninvasive tests for presence of cirrhosis and of liver function), guides treatment decisions [30]. HBeAg may also be useful to assess for viraemic HBV infection, especially where HBV DNA testing is unavailable and where use of TDF prophylaxis to prevent mother-to-child transmission in mothers with high levels of viraemia may be indicated. However, there is a paucity of good quality HBsAg and HBeAg RDTs on the market, and to date none have met the requirements for WHO prequalification [38"]. In addition, further studies are needed to validate the accuracy of HBeAg as a measure of viraemic infection compared with HBV DNA, given the lack of correlation in some studies [39].

\section{Hepatitis $C$ virus testing}

The principal assays used to determine exposure to $\mathrm{HCV}$, (indicative of either past or current infection), rely on detection of antibodies to HCV using relatively inexpensive serological assays. Currently, two RDTs have been WHO prequalified [38"]. However, such antibody-based assays are unable to detect infection soon after acquiring $\mathrm{HCV}$ infection, as antibodies may not be detected for $2-3$ months in an individual who has been recently infected. This diagnostic window period can be shortened by using assays that also directly detect HCV antigen. Once exposure to $\mathrm{HCV}$ is established, testing for viraemic $\mathrm{HCV}$ infection is performed through detection of HCV RNA [using either quantitative or qualitative nucleic acid tests (NAT)] or HCV (p22) core antigen (HCVcAg).

\section{With HIV coinfection}

There are several issues specific to diagnosis of hepatitis B and C in the context of HIV coinfection. Studies show a reduced diagnostic performance and sensitivity of HBsAg assays in HIV-infected persons [40] but that EIAs perform well. In individuals with known HIV-positive status, the pooled clinical sensitivity of RDTs was $72.3 \%$ (95\% CI: 67.976.4 ), but specificity was $99.8 \%$ (95\% CI: $99.5-$ 99.9), compared with a pooled clinical sensitivity and specificity of $92.6 \%$ (95\% CI: 89.8, 94.8) and 99.6\% (95\% CI: 99, 99.9), respectively, among HIVnegative persons. Possible explanations for this reduced sensitivity among HIV-infected persons include an increased incidence of occult hepatitis $B$ in HIV-positive persons or the use of TDF or lamivudine-based antiretroviral regimens, which are active also against HBV. Generally, the performance of anti-HCV RDTs is high [41], but there have been high rates of false positives and false negatives, particularly using earlier generation HCV serology assays in surveys in SSA [42]. HCV serological assay performance, especially RDTs, has not been well studied in HIV coinfection [41]. There is a need for further evaluation of the impact of HIV positivity (and also of $\mathrm{CD}^{+}$cell count, viral load and ART exposure, by regimen) on the sensitivity and specificity of all serological assays (especially RDTs) for the detection of HBsAg and anti-HCV, and to further validate EIAs and RDTs using less invasive and simpler methods of sample collection, such as oral fluid, capillary whole blood and dried blood spot (DBS) specimens. Finally, high-quality RDTs for screening of blood and blood products for transfusion, particularly in emergencies and in low-income settings, are needed, as current RDTs may not be sufficiently sensitive to be used for blood screening.

\section{GLOBAL TOOLS TO SUPPORT THE HEPATITIS RESPONSE}

New tools to support the hepatitis global response include a 2016-2020 Global Hepatitis Health Sector (GHHS) strategy with targets for testing and diagnosis [35"'], and WHO 2017 hepatitis testing guidelines for adults, adolescents and children in LMICs [37"']. 


\section{Global hepatitis health sector strategy and targets}

In response to the two World Health Assembly resolutions on viral hepatitis in 2010 and 2014 $[43,44]$, together with a growing recognition of the huge public health burden of hepatitis and opportunities for action, a GHHS strategy on viral hepatitis was developed and launched in 2016 [34]. This new strategy sets out goals for elimination of viral hepatitis as a public health threat by 2030 and outlines a series of impact and service delivery coverage targets as well as priority actions to be taken by countries and the WHO to achieve these targets. A major service delivery goal is the scale-up of diagnosis and those on treatment. For HBV infection, this means at least 30\% diagnosed by 2020 and $90 \%$ by 2030 (compared with $<1 \%$ in 2015) and a corresponding 8 million persons receiving hepatitis treatment by 2020 (5 million HBV and 3 million HCV) and 80\% of those eligible by 2030 (compared with 2015 baseline of $<1 \%$ ).

\section{Recommendations of WHO 2017 testing guidelines}

These are the first global guidelines on testing for $\mathrm{CHB}$ and HCV infections directed at LMICs [37"'] and complement published guidance by WHO on the prevention, care and treatment of CHB [30] and HCV [32] infections. The guidelines outline the public health approach to strengthening and expanding current testing practices for viral hepatitis and address what serological and virological assays to use (testing strategies), and who to test (testing approaches), as well as interventions to promote linkage to prevention and care. They are intended for use across different age groups and populations. The target audiences are national programme managers in ministries of health and healthcare providers in LMICs responsible for planning and implementing hepatitis testing, prevention, care and treatment services. The Grading of Recommendations, Assessment, Development and Evaluation system was used to categorize both the strength of recommendations as strong or conditional (based on consideration of the quality of evidence, balance of benefits and harms, acceptability, resource use and programmatic feasibility) and the quality of evidence as high, moderate, low or very low.

These guidelines also build on a decade of evolution in WHO HIV testing guidance [45] that includes provider-initiated testing guidance, service delivery guidance, couples testing and home based testing and recent guidance on self-testing. The key recommendations on who to test and testing approaches, how to test and testing strategies, and interventions to promote uptake of testing and linkage to care are summarized below, and the algorithms for diagnosis, management and monitoring are presented in Figs 1 and 2.

\section{Who to test for hepatitis $B$ virus and hepatitis $C$ virus infection: testing approaches}

The guidelines recommend to offer focused testing to individuals from populations most affected by $\mathrm{HBV}$ or $\mathrm{HCV}$ infection (i.e. who are either part of a population with higher seroprevalence or who have a history of exposure or high-risk behaviours for HBV or HCV infection), such as PWID, prisoners, MSM and persons infected with HIV. In settings with a seroprevalence of at least 2 to $5 \%$ of $\mathrm{HBsAg}$ or HCV antibody (anti-HCV), it is recommended that all adults have routine access and be offered testing (i.e. a general population testing approach) or to use 'birth cohort' testing for specific age groups with higher anti-HCV seroprevalence. These different testing approaches should make use of existing facility-based [such as antenatal clinics (ANC), HIV or TB services] or community-based testing opportunities including HIV testing programmes, but highlight that targeted approaches may be required for PWID and MSM in whom stigmatization and other factors may limit access to services for testing and treatment.

\section{How to test for hepatitis B virus and hepatitis $C$ virus infections: serological assays and testing strategies}

Overall, the guidelines recommend the use of a single quality-assured serological assay (i.e. either a laboratory-based EIA or RDT to detect HBsAg and anti-HCV). Assays used should meet minimum performance standards and preferably be delivered at the point-of-care to improve access and linkage to care and treatment.

\section{Confirming viraemic infection and monitoring for treatment response}

Following reactive anti-HCV serology, a quantitative or qualitative RNA NAT is recommended as the preferred testing strategy to diagnose viraemic infection. Detection of core HCV antigen, in which the assay has comparable clinical sensitivity with NAT technologies, may be considered as an alternative. The use of HBV DNA NAT following reactive HBsAg serology is recommended to help further guide who to treat or not treat if there is no evidence of cirrhosis and to monitor for treatment response based on existing recommendations from the 2015 WHO HBV management guidelines [30]. 


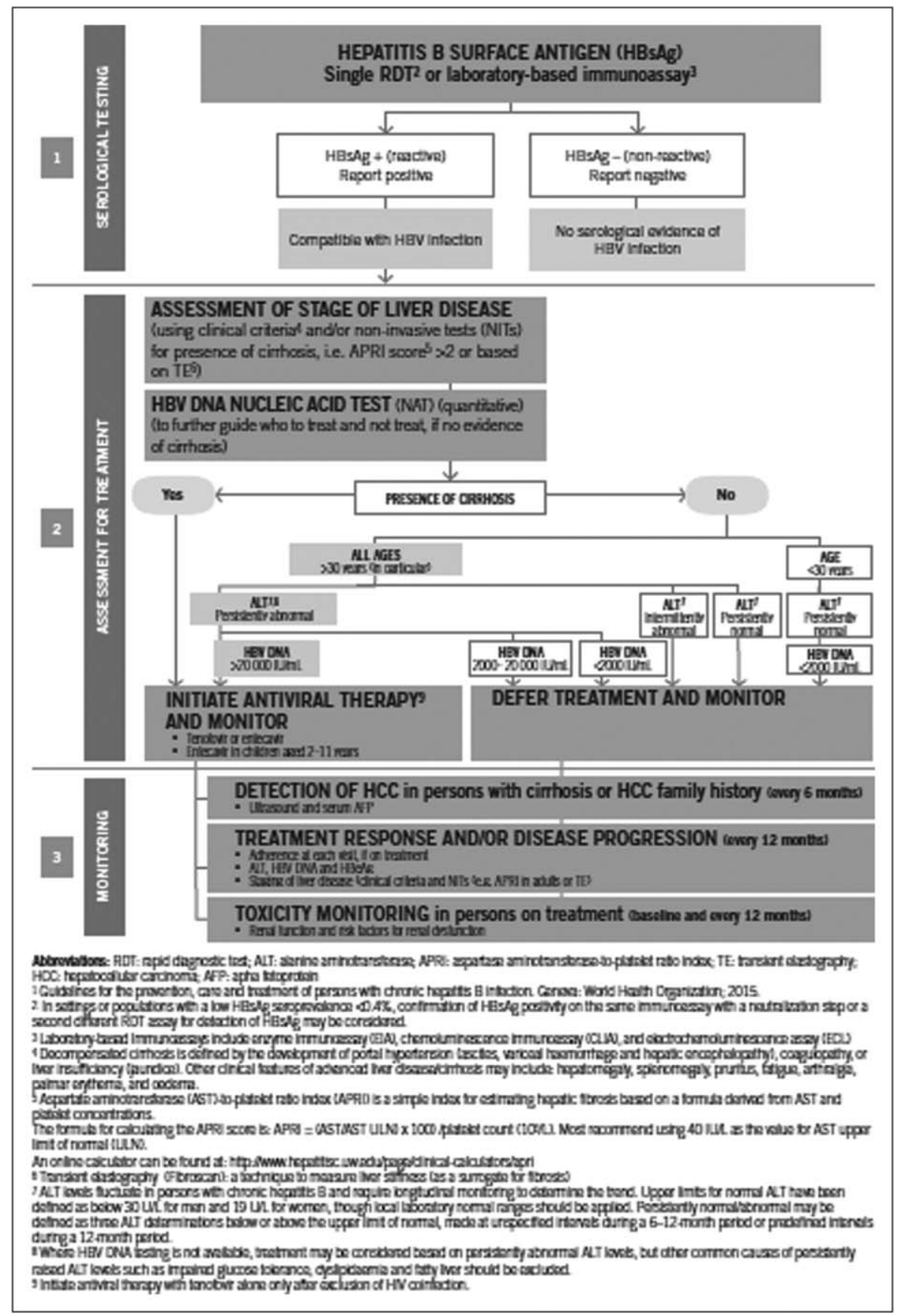

FIGURE 1. Summary algorithm for diagnosis, management and monitoring of chronic hepatitis B infection (Reproduced from $\left.\left[35^{-\prime}\right]\right)$.

\section{Use of dried blood spot specimens and other strategies to promote uptake of testing and linkage to care}

The use of capillary (finger-stick or heel-stick) whole blood DBS specimen collection for both serological and NAT assays for HBV and HCV infection may be considered in order to facilitate access to testing:- in certain settings where there are either no facilities or expertise to take venous blood specimens; in persons with poor venous access; or where quality assured
RDTs are not available or their use is not feasible. Programmes should consider only the use of assays that have been validated by their manufacturer for use with DBS specimens. Other recommended interventions to promote uptake of hepatitis testing and linkage to care include peer and lay health worker support in community-based settings, clinician reminders to offer testing in facilities, and testing as part of integrated services within drug treatment and community-based harm reduction services. 


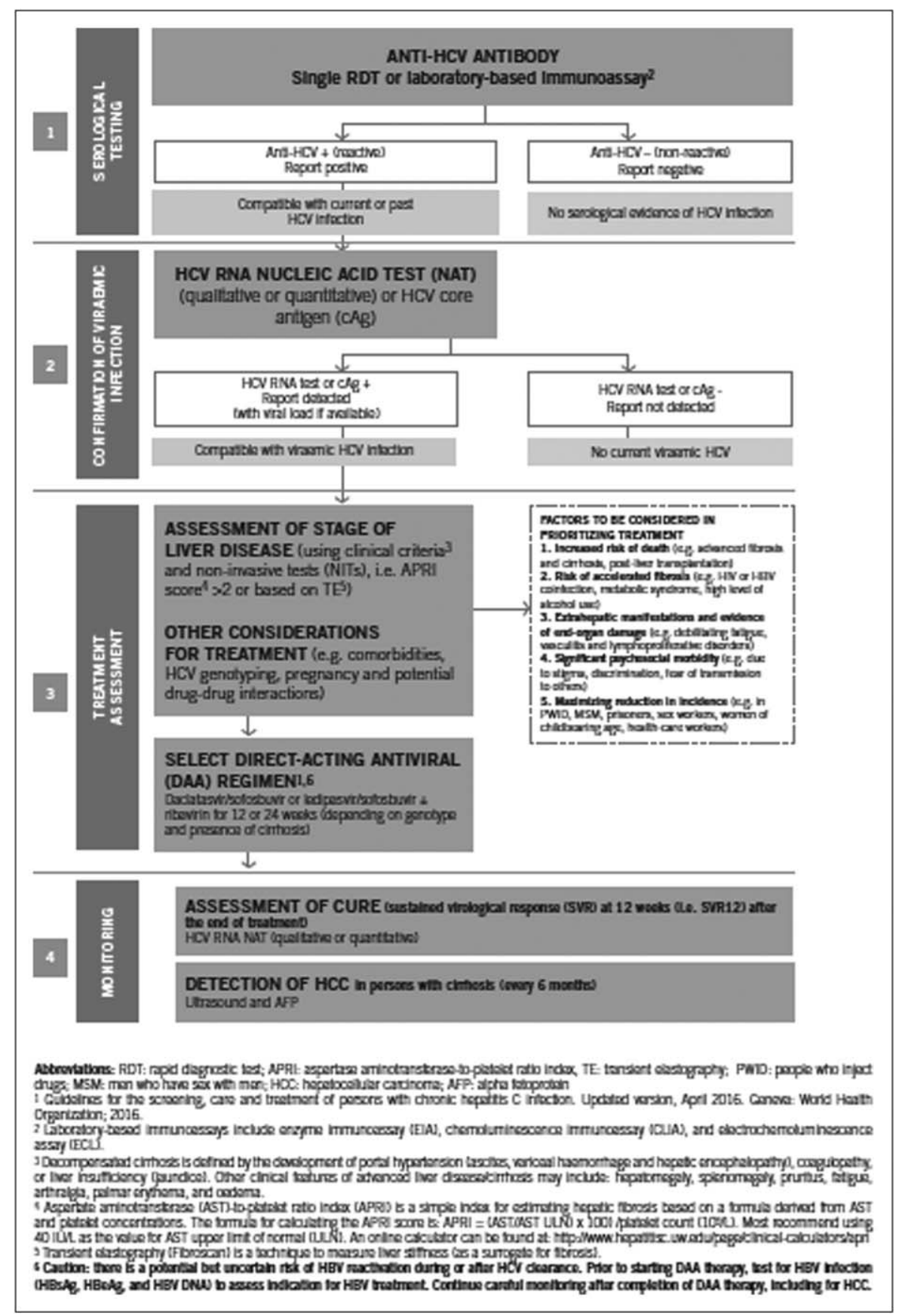

FIGURE 2. Summary algorithm for diagnosis, management and monitoring of chronic hepatitis $C$ infection (Reproduced from $\left.\left[35^{-\cdot}\right]\right)$.

\section{IMPLEMENTATION CONSIDERATIONS AND FUTURE DIRECTIONS}

\section{Guidance on implementation}

These guidelines also provide practical guidance on different service delivery models for testing, pre-test and post-test counselling and tailored testing approaches in specific populations (e.g. PWID, prisoners, pregnant women, couples and partners, children and adolescents). To support national implementation of these recommendations in LMICs, there is also a framework for country decision-making and planning in several key areas as summarized in Fig. 3 [37"'].

\section{Country requirements for implementation}

Important future directions in hepatitis testing will include the following: 
How to organise hepatitis laboratory testing services

To ensure the quality and accuracy of hepatitis testing. This includes consideration of the following key elements:

- A national framework for hepatitis testing (e.g. national testing policies, regulatory mechanism, and national reference laboratory);

- Financing strategy and planning;

- Building capacity for testing services (e.g. management of human resources, procurement and supply chain, storage and transportation, equipment and laboratory information systems);

- Setting national standards for testing (e.g. performance and operational characteristics for assays, standardized testing strategies, validation of testing algorithms (product selection), and methodology for assay selection);

- Assuring quality and safety of testing services (e.g. quality management systems, personnel, training and supportive supervision)
How to plan the best strategic mix of different testing approaches

There are many facility- and community-based approaches to delivering hepatitis testing. Countries need to consider a strategic mix of these different testing approaches to reach different populations and those currently undiagnosed, including opportunities to integrate hepatitis testing with existing services. The selection of testing approaches should be based on consideration of the following:

- National context and epidemiology (e.g. prevalence, populations affected and undiagnosed burden);

- Existing health-care and testing infrastructure and laboratory specimens testing referral network;

- Current testing uptake and coverage (number and proportion with chronic HBV or HCV who have been diagnosed $)^{3}$;

- Programme costs and cost-effectiveness of different testing approaches at national and subnational levels;

- Available financial and human resources

FIGURE 3. Implementation considerations for national hepatitis programmes. (Reproduced from [35"-]).

Service delivery models: Service delivery of hepatitis testing may be optimized by integrating hepatitis testing into other healthcare and testing services such as HIV and sexually transmitted infection clinics, antenatal services, drug dependency and prison services to increase efficiency, reach and acceptability. There is also a need to expand opportunities for community-based testing to reach marginalized groups and ensure equitable access to testing. Appropriate models of integration and linkage will depend on the country context and health system.

Adaptation of the WHO hepatitis testing guidance into national hepatitis plans and policies according to country epidemiological context and health systems that target priority populations and locations. For example, priority might be given to specific age groups (such as those born between certain dates), certain high prevalence groups (such as incarcerated persons, people who inject drugs, migrants, haemodialysis patients, people who undergo skin-piercing procedures including tattooing, some indigenous communities, sex workers and MSM) and those with evidence of liver disease.

The 3As- Accuracy, Access and Affordability represent key considerations in implementing testing services. Figure 4 illustrates the type of assays that can be deployed at different levels of the national healthcare system taking into consideration the capacity of facilities, human resources/ technical expertise and infrastructure/environmental demands (e.g. electrical, refrigeration and may involve a trade-off between accuracy and access to achieve greater coverage of testing. For example, at the community or primary healthcare level, testing must be simple and able to be performed by a community worker with use of RDTs. Countries also need to assess the costs of implementing a testing programme and determine what are the drivers of costs to maximize resources. There is a need for simple easy-to-use tools for costing and modelling to support country business plan developments.

Strengthening of national laboratory systems to provide quality diagnosis of $\mathrm{CHB}$ and $\mathrm{HCV}$ infection, and a reliable supply of quality-assured (WHOprequalified) diagnostics, with simplification of the hepatitis diagnostic pathway. There is a need for more reliable and low-cost RDTs for viral hepatitis B and $\mathrm{C}$, point-of-care (POC) testing for monitoring hepatitis B and C viral load (and hepatitis C antigen) to guide treatment decisions and simplified tests for assessing liver fibrosis and cirrhosis.

Accurate forecasting of country and global needs in diagnostic commodities. Effective testing programmes rely on an uninterrupted supply of diagnostics. Robust procurement and supply management 


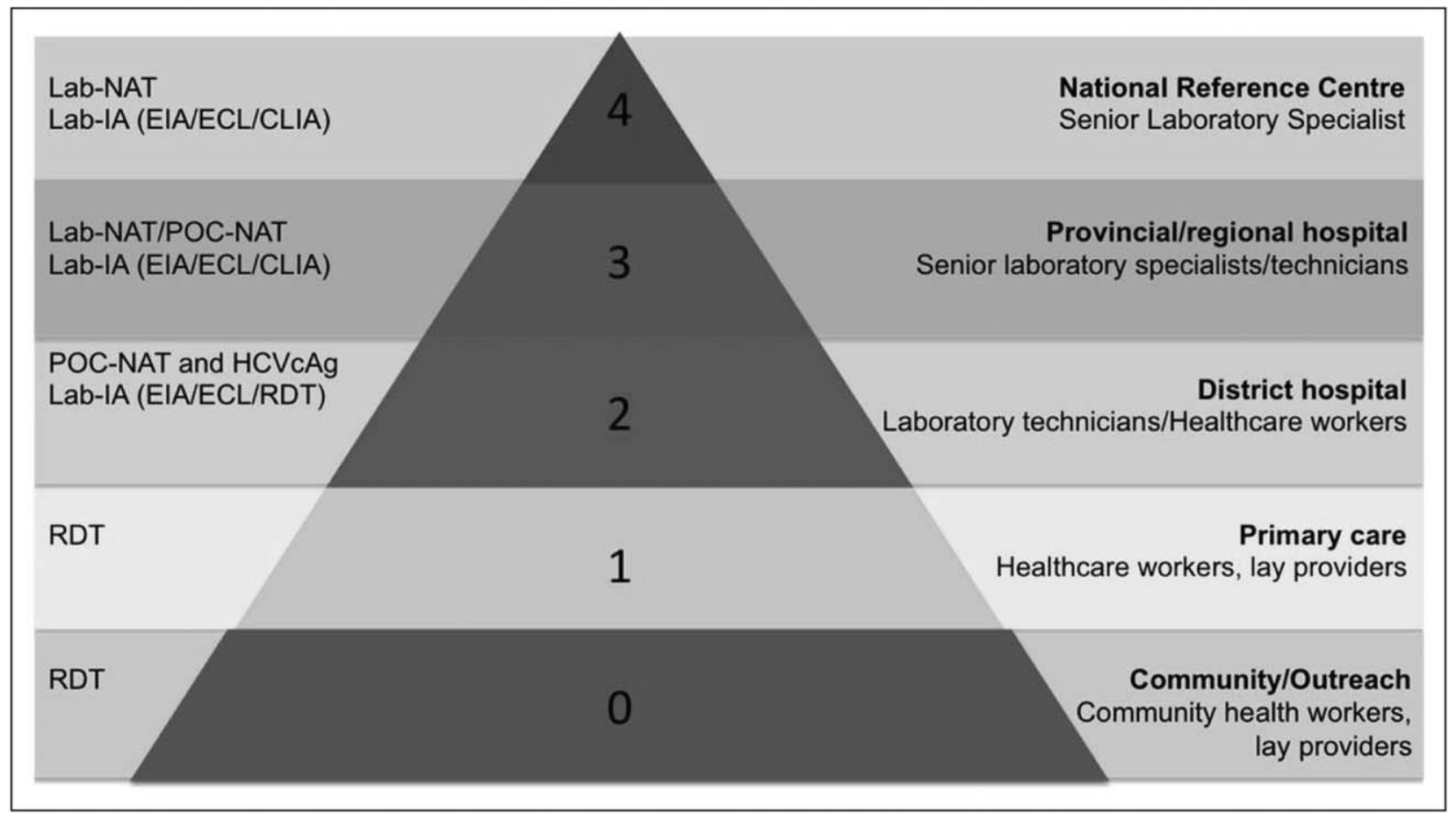

FIGURE 4. Matching diagnostic needs and capacity at different levels of the national healthcare system, with provision of different types of tests depending on the type of facility. Serology: CLIA, chemiluminescence immunoassay; ECL, electrochemiluminescence immunoassay; EIA, enzyme immunoassay; IA, laboratory-based immunoassay; RDT, rapid diagnostic test.; Virology: HCVcAg, HCV core antigen; Lab-NAT; laboratory-based NAT; NAT, nucleic acid test; POC-NAT, point-of-care NAT (Reproduced from [35"-]).

systems, such as bulk procurement with staggered deliveries, advanced purchasing and improved forecasting are required to ensure savings on procurement and introduce efficiencies in supply management.

\section{Quality assurance of hepatitis diagnostics and the WHO prequalification programme}

A key barrier to the more widespread adoption of testing is the current lack of quality-assured RDTs. An important contributor to facilitating access to invitro diagnostics (IVDs) of good quality and building the capacity of national authorities to more effectively monitor quality of IVDs is the WHO Prequalification of Diagnostics programme that includes hepatitis B and C diagnostics $\left[38^{*}, 46\right]$. Only a third of countries have some type of regulatory system in place for IVDs; and even where regulations for IVDs exist, often they are not enforced. WHO specifically targets prequalification of the type of IVDs more commonly used in resource-limited settings such as RDTs and EIAs without automated test procedures, which are generally not used in high-income countries where sophisticated centralized laboratory infrastructure exist.

The current WHO prequalification pipeline for hepatitis diagnostic assays as of December 2016 includes eight HCV IVDs and two HBsAg IVDs that are considered eligible to proceed for WHO prequalification according to standardized procedures. To date, two anti-HCV RDTs, two anti-HCV EIAs, one anti-HCV line immunoassy, four HBsAg EIAs have been WHO prequalified. RDTs with a high degree of ease of use that can be used with capillary (fingerstick) whole blood with reduced requirement for cold chain storage will be prioritized given their unique applicability in resource-limited settings where infrastructure and human resources are lacking.

\section{DIAGNOSTIC INNOVATIONS AND FUTURE DEVELOPMENTS}

Advances in hepatitis virus detection technology have also created new opportunities for enhancing hepatitis testing, as well as monitoring of response to treatment [42]. Future directions and innovations in testing include simplified single virological assay testing strategies, assays for near-patient or POC use for NAT and core antigen, DBS, multiplex and multidisease platforms and self-testing for anti-HCV.

\section{Simplified testing strategies}

Simplifying testing algorithms will be critical to ensuring affordability and the success of scaling 
up testing. Potential future testing approaches for HCV infection are the adoption of a lessexpensive and more manageable single virological tests for both diagnosis and confirmation of viraemia $[47,48]$. However, this may only ever be costeffective in high-prevalence settings and high-risk populations.

\section{Near-patient or point-of-care testing}

The development of reliable, accurate, practical and affordable assays that may be used at or near to POC will be crucial for expanding hepatitis testing services, especially in community-based settings, where there are challenges with cost, transport and venepuncture requirements [49]. These technologies for viral hepatitis include molecular NAT-based tests for diagnosis and treatment monitoring and HCVcAg testing. These emerging POC devices are able to perform conventional laboratory molecular testing (qualitative and quantitative) in field settings; are easier to use than the laboratory-based NAT assays, as they require minimum training; can be operated on battery or conventional power source; do not require phlebotomy and provide a result within $2 \mathrm{~h}$. Among these are cartridge-based HCV RNA assays, which can be used with existing diagnostic platforms developed for tuberculosis or HIV early infant diagnosis and viral load monitoring. There are also HCVcAg POC platforms in development. These devices offer the possibility of a same-day diagnosis of viraemic infection, either alone or when combined with an HCV antibody RDT, and for test of cure. Outstanding research questions include establishing the distribution of HCV viral load at diagnosis and in the context of viral rebound with treatment failure [and, specifically, the proportion of patients with low viral loads missed by assays that have higher limits of detection (e.g. $3000 \mathrm{IU} / \mathrm{ml})$ ]. This information will be useful in assessing the clinical utility of HCV qualitative versus quantitative RNA assays and optimizing the development of future HCV RDTs for HCVcAg detection, including as a one-step diagnostic strategy in a range of higher prevalence settings. A quantitative HBsAg test is currently under evaluation as a potentially simplified alternative strategy to ascertain high levels of viraemia, rather than a test for HBeAg or HBV DNA [50].

\section{Dried blood spot specimens}

Validation of existing assays that can be used with DBS for serological diagnosis, as well as detection and quantification of HCV RNA and HBV DNA, show promise for extending access to hepatitis testing especially in community-based settings. Two recent reviews showed good diagnostic performance using DBS sampling with different commercial hepatitis serological and NAT assays [51,52]. DBS has been used extensively for early infant diagnosis of HIV but the quality of DBS specimens varies widely due to different collection, drying and storage and elution methods. However, one major challenge in programmes using DBS is linkage to care. Although DBS can be collected at remote sites, specimens have to be sent off to centralized laboratories for testing, often creating major delays in result reporting. Priority areas for research and development include DBS specimen validation by manufacturers of commercial serological and NAT assays, and application for stringent regulatory approval and WHO prequalification to use DBS as an additional specimen type.

\section{Multiplex and multi-disease analysers}

Multiplex and multi-disease analysers allow for integrated testing of hepatitis $\mathrm{B}$ and $\mathrm{C}$ alongside other pathogens, for example HIV and syphilis, and can leverage technology developed for other infectious disease programmes. Key advantages include the requirement for lower specimen volume, improved client flow with results for multiple pathogens available at the same time and so fewer patient visits and transport costs. Multiplex RDTs are in development for anti-HIV/antiHCV, anti-HIV/syphilis/anti-HCV, anti-HIV/syphilis/HBsAg and anti-HIV/anti-HCV/HBsAg. Data on their diagnostic accuracy and impact on patientimportant outcomes are required before adoption. Typically, they are most suitable for district, provincial and national laboratories as the current analysers may require certain precision steps, utilize serum/ plasma specimens and require constant electricity supply and regular maintenance.

\section{Self-testing}

Self-testing is a process in which an individual, who wants to know his or her status, collects a specimen, performs a test and interprets the result themselves, often in private. HIV self-testing is now being conducted in many settings and has increased uptake of testing among people not reached by other existing HIV testing services, many of whom are first-time testers [53]. The experience with hepatitis self-testing is currently very limited, but it represents a potentially important approach to expand access to testing in the future. 


\section{Service delivery}

In addition to these technological innovations, there have been advances in the mode of delivery of testing, so that promising new technologies reach target populations. Supply chain management is one of the major challenges in healthcare delivery in low-income rural settings where there is limited access to laboratories or any form of diagnostic testing. Although assays for use at POC can help increase access to hepatitis testing, such decentralization of testing can also place major stresses on already fragile health systems. Unmanned aerial vehicles (UAVs), also referred to as drones, are remotely operated robotic airplanes that have emerged as a potentially valuable technology to deliver healthcare supplies, such as RDTs, DBS specimens, blood products and vaccines, to remote locations with poor transport routes, especially during the rainy season [53]. UAVs can carry a payload of $2-3 \mathrm{~kg}$ (sufficient for 100 RDTs), fly a distance of approximately $60 \mathrm{~km}$ and either land or do aerial drops of their payload onto a target area. Pilot projects in HIV and other areas are ongoing in Malawi, Rwanda, Zambia, the Dominican Republic, Haiti, Papua New Guinea and Bhutan [54-57].

A further priority is to address key research gaps that will in turn inform future global guidance on viral hepatitis testing in LMICs. In particular, there is a need for formal evaluation of different HBV and HCV testing approaches [i.e. routine general population, focused risk-based, antenatal clinic (ANC) and birth cohort testing] using different service delivery models (community-facility-based or health-facility-based). This can take the form of comparative trials, large-scale implementation studies or demonstration projects in a range of epidemic settings and populations in LMICs.

\section{CONCLUSION}

To overcome current challenges in hepatitis testing and substantially increase awareness of hepatitis status and earlier diagnosis, there is a need to provide a secure supply of quality-assured affordable diagnostics, services that can reach those populations most affected, well functioning laboratories to ensure high-quality testing and treatment monitoring, an appropriately trained health workforce and active involvement of affected communities. The new WHO global testing guidelines provide a major opportunity to improve identification and treatment of persons with $\mathrm{CHB}$ and $\mathrm{HCV}$ infection, and achieve the 2016 Global Health Sector Strategy on viral hepatitis targets on testing and treatment. This will, in turn, improve clinical outcomes, save lives and reduce hepatitis B and C transmission. Advances in hepatitis virus detection technology have created new opportunities for enhancing testing referral and treatment, with simplified one-assay testing algorithms, use of POC molecular tests, multiplex or polyvalent testing, self-testing and new modes of service delivery.

\section{Acknowledgements}

Many professionals from a range of backgrounds and specialties have contributed to the development of this guidance. WHO is sincerely grateful for their time and support. The following experts served on the Guidelines Development Group: Jacinto Amandua (Ministry of Health, Uganda); Isabelle Andrieux-Meyer (Médecins Sans Frontières, Geneva, Switzerland), Manal Hamdy El-Sayed (Egypt National Hepatitis Committee), Charles Gore (World Hepatitis Alliance, London, UK), Niklas Luhmann (Médicins du Monde, Paris, France), Michael Ninburg (Hepatitis Education Project, Seattle, USA), Richard Njouom (Centre Pasteur of Cameroon), John Parry (Public Health England, London, UK), Trevor Peter (Clinton Health Access Initiative, New York, USA), Teri Roberts (Foundation for Innovative New Diagnostics, Geneva, Switzerland), Giten Khwairakpram Singh (TREAT Asia/amFAR), Lara Tavoschi (European Center for Disease Prevention and Control, Stockholm, Sweden), Roger Chou (Oregon Health \& Science University, Portland, USA)(Methodologist) and Richard Tedder - unable to attend (Public Health England, London, UK). The chair of the Guidelines Development Group was Margaret Hellard (Burnet Institute, Melbourne Australia). Roger Chou (Oregon Health and Science University, Portland, USA) was the guidelines methodologist. WHO Regions: Fabian Ndenzako (WHO Regional Office for Africa), Nicole Simone Seguy (WHO Regional Office for the South East Asia) and Nick Walsh (WHO Regional Office for the Western Pacific).

The opinions expressed are those of the author(s) and do not necessarily represent those of the organization.

\section{Financial support and sponsorship}

None.

\section{Conflicts of interest}

There are no conflicts of interest.

\section{REFERENCES AND RECOMMENDED}

\section{READING}

Papers of particular interest, published within the annual period of review, have been highlighted as:

- of special interest

-n of outstanding interest

1. World Health Organization. Global health sector response to HIV, 20002015: focus on innovations in Africa: progress report. Geneva: World Health Organization; 2016. 
2. Stover J, Andreev K, Slaymaker E, et al. Updates to the Spectrum model to estimate key HIV indicators for adults and children. AIDS 2014; 28:S427S434.

3. Schweitzer A, Horn J, Mikolajczyk RT, et al. Estimations of worldwide

- prevalence of chronic hepatitis B virus infection: a systematic review of data published between 1965 and 2013. Lancet 2015; 386:15461555.

This systematic review provides updated regional and global estimates of prevalence and burden of chronic hepatitis B infection.

4. Gower E, Estes C, Blach S, et al. Global epidemiology and genotype distribution of the hepatitis $C$ virus infection. J Hepatol 2014; 61 (1 Suppl): S45-S57.

5. Basnayake SK, Easterbrook PJ. Wide variation in estimates of global prevalence and burden of chronic hepatitis $B$ and $C$ infection cited in published literature. J Viral Hepatitis 2016; 23:545-559.

6. GBD 2013 Mortality and Causes of Death Collaborators. Global, regional and national age specific all-cause and cause-specific mortality for 240 causes of death 1990-2013: a systematic analysis for the Global Burden of Disease Study 2013. Lancet 2015; 385:117-171.

7. Lok AS, McMahon BJ. Chronic hepatitis B. Hepatology 2007; 45:507539.

8. Shepard CW, Finelli L, Alter MJ. Global epidemiology of hepatitis C virus infection. Lancet Infect Dis 2005; 5:558-567.

9. Easterbrook $P$, Sands A, Harmanci $H$. Challenges and priorities in the management of HIV/HBV and HIV/HCV coinfection in resource-limited settings. Semin Liver Dis 2012; 32:147-157.

10. Sulkowski MS. Viral hepatitis and HIV coinfection. J Hepatol 2008; 48:353367.

11. Puoti M, Spinetti A, Ghezzi A, et al. Mortality for liver disease in patients with HIV infection: a cohort study. J Acquir Immune Defic Syndr 2000; 24:211217.

12. Peters $\mathrm{L}$, Klein MB. Epidemiology of hepatitis $\mathrm{C}$ virus in HIV-infected patients. Curr Opin HIV AIDS 2015; 10:297-302.

13. Weber R, Sabin C, Friis-Møller N, et al. Liver-related deaths in persons infected with the HIV: the D:A:D study. Arch Intern Med 2006; 166:1632-1641.

14. Nikolopoulos GK, Paraskevis D, Hatzitheodorou E, et al. Impact of hepatitis B virus infection on the progression of AIDS and mortality in HIV-infected individuals: a cohort study and meta-analysis. Clin Infect Dis 2009; 48:1763-1771.

15. Easterbrook PJ, Platt L, Gower E et al. Global systematic review and meta-

- analysis of the seroprevalence of HBV and HCV infection in HIV-infected persons. 8th IAS Conference on HIV Pathogenesis, Treatment and Prevention. 19-22 July 2015. Abstract no. TU PEB254.

This systematic review and meta-analysis provides new regional and global estimates of prevalence and burden of HIV-hepatitis B virus (HIV-HBV) coinfection in five different population groups and highlights important distinctions between epidemiology of HIV-HBV and HIV-hepatitis C virus (HIV-HCV) coinfection.

16. Platt L, Easterbrook PJ, Gower E, et al. Prevalence and burden of HCV co-

- infection among people living with HIV: a global systematic review. Lancet Infect Dis 2016; 16:797-808.

This systematic review and meta-analysis provides new regional and global estimates of prevalence and burden of HIV-HCV coinfection in five different population groups.

17. Stabinski L, O'Connor S, Barnhart M, et al. Prevalence of HIV and hepatitis B virus co-infection in sub-Saharan Africa and the potential impact and program feasibility of hepatitis B surface antigen screening in resource-limited settings. J Acquir Immune Defic Syndr 2015; 68 (Suppl 3):S274-S285.

18. Thio CL. 2009 Hepatitis $B$ and human immunodeficiency virus coinfection. Hepatology 2009; 49 (5 Suppl):S138-S145.

19. Barth RE, Huijgen Q, Taljaard J, Hoepelman Al. Hepatitis B/C and HIV in subSaharan Africa: an association between highly prevalent infectious diseases. A systematic review and meta-analysis. Int J Infect Dis 2010; 14:e1024e1031.

20. Rao VB, Johari $N$, du Cros $P$, et al. Hepatitis $C$ seroprevalence and HIV coinfection in sub-Saharan Africa: a systematic review and meta-analysis. Lancet Infect Dis 2015; 15:819-824.

21. Benova L, Mohamoud YA, Calvert C, Abu-Raddad LJ. Vertical transmission of hepatitis C virus: systematic review and meta-analysis. Clin Infect Dis 2014; 59:765-773.

22. Floreani A. Hepatitis C and pregnancy. World J Gastroenterol 2013; 19:6714-6720.

23. Valle Tovo C, Alves de Mattos A, Ribeiro de Souza A, et al. Impact of human immunodeficiency virus infection in patients infected with the hepatitis $\mathrm{C}$ virus. Liver Int 2007; 27:40-46.

24. Thomas DL, Astemborski J, Rai RM, et al. The natural history of hepatitis $C$ virus infection: host, viral, and environmental factors. JAMA 2000; 284:450456.

25. Greub G, Ledergerber B, Battegay $M$, et al. Clinical progression, survival, and immune recovery during antiretroviral therapy in patients with HIV-1 and hepatitis C virus coinfection: the Swiss HIV Cohort Study. Lancet 2000; 356:1800-1805.
26. Zollner B, Petersen J, Puchhammer-Stockl E, et al. Viral features of lamivudine resistant hepatitis B genotypes A and D. Hepatology 2004; 39:42-50.

27. Nelson NP, Easterbrook PJ, McMahon BJ. Epidemiology of hepatitis B infection and impact of vaccination on disease. Clin Liver Dis 2016; 20:607-628.

28. WHO/UNICEF estimates of National Immunization coverage 2016. Available at: www.who.int/immunization/monitoring_surveillance/routine/coverage/.../ index4.html. [Accessed 26 February 2017]

29. Consolidated guidelines on HIV diagnosis, prevention and treatment among key populations. Geneva: World Health Organization; 2014.

30. World Health Organization. Guidelines for the prevention, care and treatment of persons with chronic hepatitis B infection. Geneva: World Health Organization; 2015.

31. World Health Organization. Consolidated guidelines on the use of antiretroviral drugs for treating and preventing HIV infection. Geneva: World Health Organization; 2016.

32. World Health Organization. Guidelines for the screening, care and treatment of persons with chronic hepatitis, C infection. Geneva: World Health Organization; 2016.

33. Chang ML, Liaw YF. Hepatitis B flares in chronic hepatitis B: pathogenesis, natural course, and management. J Hepatol 2014; 61:1407-1417.

34. Coffie PA, Egger M, Vinikoor MJ, et al. Trends in viral hepatitis B screening practices and management in HIV clinics across sub-Saharan Africa, 20102012. BMC Infect Dis 2017. (in press).

35. World Health Organization. WHO global health sector strategy on viral

mepatitis. Geneva: World Health Organization; 2016 ; http://www.who.int/ hepatitis/strategy2016-2021/. [Accessed 09 January 2017]

First global strategy on hepatitis that sets out a series of impact and service delivery targets as well as priority actions for countries and WHO to achieve these.

36. Easterbrook P, Johnson C, Figueroa C, Baggaley R. HIV and hepatitis testing: global progress, challenges and future directions. AIDS Rev 2016;18:3-14

37. World Health Organization. Guidelines on hepatitis B and C testing. Geneva:

* World Health Organization; 2017.

New evidence-based global guidelines on who to test and how to test for chronic hepatitis $B$ and $C$, and strategies for implementation.

38. Status of $\mathrm{WHO}$ prequalification of hepatitis diagnostics. Available at: http:// www.who.int/diagnostics_laboratory/evaluations/pq-list/hcv/public_report/ en. [Accessed 26 February 2017]

Website provides updated information on status of quality assessments of hepatitis $\mathrm{B}$ and $\mathrm{C}$ assays for $\mathrm{WHO}$ prequalification.

39. Shimakawa $Y$, Toure-Kane C, Mendy M, et al. Mother-to-child transmission of hepatitis B in sub-Saharan Africa. Lancet Infect Dis 2016; 16:19-20.

40. Amini $A$, Varsaneux $O$, Kelly $H$, et al. Diagnostic accuracy of tests to detect Hepatitis B surface antigen: a systematic review of the literature and metaanalysis. BMC Infect Dis 2017. (in press).

41. Tang $W$, Chen $W$, Amini $A$, et al. Diagnostic accuracy of tests to detect hepatitis c antibody: a meta-analysis and review of the literature. BMC Infect Dis 2017. (in press).

42. Callahan JD, Constantine NT, Kataaha $P$, et al. Second generation hepatitis $C$ virus assays: performance when testing African sera. J Med Virol 1993; $41: 35-38$

43. World Health Organization. Sixty-third world health assembly. Resolution WHA63. 18 on viral hepatitis. Geneva: World Health Organization; 2010; http://apps.who.int/gb/ebwha/pdf_files/WHA63-REC1/WHA63_REC1-en.pdf. [Accessed 20 June 2016]

44. World Health Organization. Sixty-seventh world health assembly. Resolution WHA67. 6 on hepatitis. Geneva: World Health Organization; 2014; http:// apps.who.int/gb/ebwha/pdf_files/WHA67/A67_R6-en.pdf. [Accessed 20 June 2016]

45. World Health Organization. WHO consolidated guidelines on HIV testing. Geneva: World Health Organization; 2016.

46. World Health Organization. Guidance for procurement of in vitro diagnostics and related laboratory items and equipment. Geneva: WHO; 2013; http:// www.who.int/diagnostics_laboratory/procurement/131024_procurement_of_ diagnostics_finalversion.pdf?ua=1. [Accessed 02 July 2016]

47. Cohn J, Roberts $T$, Amorosa V, et al. Simplified diagnostic monitoring for hepatitis $\mathrm{C}$, in the era of direct acting antiviral treatment. Curr Opin HIV AIDS 2015; 10:369-373.

48. WHO/UNITAID. Hepatitis $C$ medicines and diagnostics in the context of HIV/HCV co-infection: a scoping report. Geneva: WHO/UNITAID; 2013.

49. World Health Organization. The quality of HIV-related point-of-care testing: ensuring reliability and accuracy of test results. Geneva: World Health Organization; 2015; http://www.who.int/hiv/pub/toolkits/handbook-point-ofcare-testing/en/. [Accessed 02 July 2016]

50. Dusheiko G, Easterbrook $P$. Bringing to an end mother to child transmission of hepatitis B: a role for quantitative HBsAg? Hepatology 2016; 64:14081410; Editorial.

51. Lange B, Cohn J, Roberts $T$, et al. Diagnostic accuracy of serological diagnosis of hepatitis $\mathrm{C}$ and $\mathrm{B}$ using dried blood spot samples: two systematic reviews and meta-analyses. BMC Infect Dis 2017. (in press). 
52. Lange $B$, Roberts $T$, Cohn J, et al. Detection and quantification of HBV DNA and HCV RNA using dried blood spot (DBS) samples - a systematic review and meta-analysis. BMC Infect Dis 2017. (in press).

53. World Health Organization. Guidelines on HIV self-testing and partner notification: supplement to consolidated guidelines on HIV testing services. Geneva: World Health Organization; 2016.

54. Amukele $T$, Ness $P M$, Tobian $A A$, et al. Drone transportation of blood products. Transfusion; 2016. doi: 10.1111/trf.13900.
55. Drones are revolutionising healthcare in Madagascar. http://www.digital trends. com/cool-tech/drones-healthcare-madagascar/. [Accessed 07 January 2017]

56. How Rwanda is using drones to save millions of lives. http://www.cnbc.com/ 2016/05/27/how-rwanda-is-using-drones-to-save-millions-of-lives.html. [Accessed 07 January 2017]

57. The Government of Malawi and UNICEF announce first humanitarian drone testing corridor in Africa. Dec 2016. https://www.unicef.org/media/media 94184.htm. [Accessed 07 January 2017] 